\title{
The case of the oscillating party balloon: A simple toy experiment requiring a not-so-simple interpretation
}

\author{
Amnon Yariv \\ Department of Applied Physics, California Institute of Technology, Pasadena, California 91125 \\ Jacob Scheuer ${ }^{a}$ \\ School of Electrical Engineering, Tel-Aviv University, Ramat-Aviv, Tel-Aviv, Israel 69978 \\ Bruno Crosignani and Paolo Di Porto \\ Dipartimento di Fisica, Universita' dell'Aquila, L'Aquila, Italy and Research Center Soft INFM-CNR \\ clo Universita' di Roma, "La Sapienza," Rome, Italy
}

(Received 29 November 2006; accepted 30 March 2007)

\begin{abstract}
The intriguing midair oscillations of a party balloon, which occur once its buoyancy is no longer capable of keeping it against the ceiling, is shown to require a rather sophisticated explanation in terms of variable-mass dynamics. The ubiquity of this phenomenon, the accessibility of its actual observation, and the subtlety of its analytic description provide a good opportunity for an interesting zero-cost classroom demonstration. (C) 2007 American Association of Physics Teachers.
\end{abstract}

[DOI: 10.1119/1.2733684]

\section{INTRODUCTION}

Under usual circumstances, metallic party balloons do not offer an occasion for any profound thought about their behavior. They move, under the combined influence of gravity and Archimedes' force, toward the ceiling of the room. However, if one can observe them long enough, a new scenario emerges: suddenly, one of the balloons begins to descend slowly until, after a while, the descent stops and the balloon reverses its direction of motion only to reverse it again at a certain height and so on. This behavior consists of a damped vertical oscillatory motion around a midair equilibrium position at which the balloon eventually comes to rest.

Our intuition immediately associates the oscillatory motion with the presence of the balloon tail (see Fig. 1), which is responsible for a variable-mass dynamics. At a given time, the buoyancy of the metallic balloon (which remains approximately constant over a long period of time) is no longer able to overcome gravity. At this time, the system consisting of the balloon proper plus its tail begins to descend and, in so doing, loses the fraction of its weight corresponding to the part of the tail which lands on the floor. The balloon descends until Archimedes' force becomes dominant again, the motion is reversed, and the mass of the system increases up to a height at which the motion is reversed again. We can conjecture that some kind of dissipation mechanism, such as air resistance, eventually brings the oscillatory motion to a stop in an equilibrium position. In practice, the phenomenon is usually observed by slightly displacing the balloon, upward or downward, from its equilibrium position.

The behavior of the balloon can be quantitatively described by the simple model that will be introduced in this paper. This deceivingly simple problem presents some intriguing features associated with the nonanalytic behavior of its solution, which is produced by the interaction of the tail with the ground. In particular, even if we neglect air resistance when appropriate, we can show an energy-dissipating process to be inherently present. This dissipation, due to the transfer of the tail momentum to the floor during the downward motion and to the work done by the tail tension during the upward motion, has a simple analytic expression and represents a new kind of oscillation-damping mechanism.
Even though lighter-than-air balloons have been around for a long time, this particular kind of problem seems to have been almost completely overlooked. The dynamics of a balloon-payload system was studied in Ref. 1, and the oscillations of a gas balloon due to temperature gradients were studied in Ref. 2. A configuration similar to ours was discussed in Ref. 3. Only the equilibrium situation was considered and the possibility of oscillations was only briefly mentioned.

\section{VARIABLE-MASS DYNAMIC BEHAVIOR OF THE BALLOON}

Our system consists of a metallic balloon of radius $\mathrm{R}$, containing a light gas (helium), tied to a string of linear mass density $\lambda$ (the tail) hanging under the balloon and partially touching the ground (see Fig. 1). For simplicity, we assume that the tail is in constant contact with the ground (while the balloon itself never touches it), and indicate by $x(t)>0$ the distance between the ground and the bottom of the balloon, so that $m(t)=\lambda x(t)$ is the mass of the vertical string portion.

For upward motion, the total external force is $F-M g$ $-\lambda x g+F_{a}$, where $F$ is the Archimedes force, $M$ is the mass of the balloon and the interior gas, and $F_{a}$ is the air resistance. In particular, for the range of Reynolds numbers that apply to our experiment, the viscous drag term linear in $\dot{x}$ is negligible and $\left|F_{a}\right|=\rho_{a} C \dot{x}^{2} A / 2$, where $\rho_{a}$ is the air density, $A$ is the transverse area of the balloon, and $C$ is the drag resistance coefficient, which depends on the body shape. As a consequence, Newton's second law reads

$$
F-M g-\lambda g x-\hat{\lambda} \dot{x}^{2}=\frac{d}{d t}[(M+\lambda x) \dot{x}]=(M+\lambda x) \ddot{x}+\lambda \dot{x}^{2},
$$

where we have introduced the quantity $\hat{\lambda}=\rho_{a} A C / 2$ (which has the dimensions of a linear mass density).

During the downward motion, the string element of length $-\dot{x} \Delta t$ touching the floor in the time interval $\Delta t$ comes to a stop, which amounts to a totally inelastic collision (zero restitution coefficient). This stop corresponds to a momentum variation $\Delta p=\lambda \dot{x}^{2} \Delta t$, which results in a dissipative force 


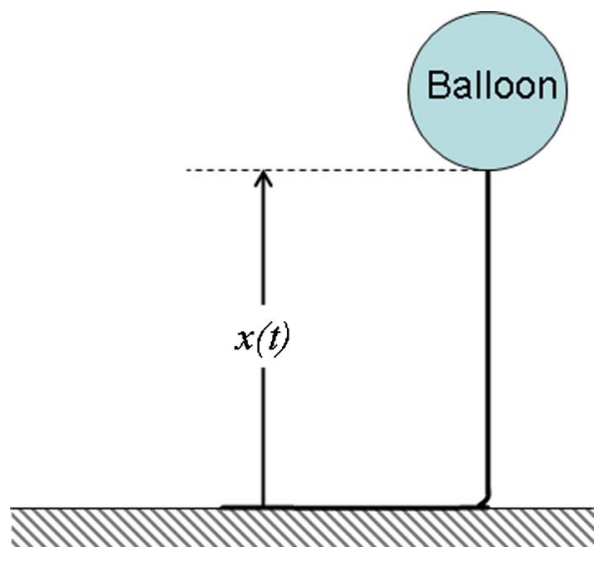

Fig. 1. The balloon-string system.

$F_{0}=\Delta p / \Delta t=\lambda \dot{x}^{2}$ exerted by the floor. Therefore, the equation of motion differs from Eq. (1a) by the additional term $F_{0}$ on the left-hand side, which cancels the $\lambda \dot{x}^{2}$ term on the righthand side of Eq. (1a), leading to

$$
F-M g-\lambda g x+\hat{\lambda} \dot{x}^{2}=(M+\lambda x) \ddot{x} .
$$

Equations (1a) and (1b) must be solved piece-wise and the relative solutions matched when $\dot{x}$ vanishes.

Even without explicit integration, Eqs. (1a) and (1b) yield an analytic expression for the energy dissipation rate. The total mechanical energy of the system is $E=T+V$, where $T$ $=\left(\lambda x \dot{x}^{2}+M \dot{x}^{2}\right) / 2$ is the kinetic energy and $V=-F x+M g x$ $+\lambda g x^{2} / 2$ is the potential energy associated with the (conservative) gravitational and Archimedes' forces. The time derivative of $E$ yields, with the help of Eqs. (1a) and (1b),

$$
\begin{aligned}
& \frac{d E}{d t}=-\left(\frac{1}{2} \lambda+\hat{\lambda}\right) \dot{x}^{3} \quad \text { (upward motion), } \\
& \frac{d E}{d t}=\left(\frac{1}{2} \lambda+\hat{\lambda}\right) \dot{x}^{3} \quad \text { (downward motion). }
\end{aligned}
$$

Equations (2a) and (2b) express the existence of a new power-dissipation mechanism during the upward and downward motion of the system, together with that due to air resistance.

\section{THE ORIGIN OF DISSIPATION (OTHER THAN AIR RESISTANCE)}

Energy dissipation can be given a simple interpretation in terms of the external work performed by the floor on the small part of the tail reaching it during a time interval $\Delta t$ (downward motion) and of the internal work performed by the tension $\tau$ on the small part of the tail leaving the floor in the time interval $\Delta t$ (upward motion).

During the upward motion the tension exerted by the bottom of the string on the small part of the tail leaving the floor in a time interval $\Delta t$ produces an increase $\Delta p$ of its momentum given by

$$
\Delta p=\dot{x} \Delta m=\lambda \dot{x}^{2} \Delta t,
$$

so that

$$
\tau=\frac{\Delta p}{\Delta t} \underset{\Delta t \rightarrow 0}{=} \frac{d p}{d t}=\lambda \dot{x}^{2} .
$$

In the same interval $\Delta t$ a small part of the string goes from a vanishing length (when lying loosely on the floor) to a length $\Delta x$ so that the "head" of the small part undergoes a displacement $\Delta x$, while its "bottom" undergoes no vertical displacement: it is as if the small part of the string undergoes on the average an internal length increase $\Delta x / 2$. Thus, the tension at $x=0$ performs both a positive work $\tau \Delta x / 2$ on the tail leaving the floor and a negative work $-\tau \Delta x$ on the vertical tail part. As a consequence, we have a total negative work $\Delta W_{\text {up }}$ given by

$$
\Delta W_{\text {up }}=-\tau \Delta x / 2=-\lambda \dot{x}^{3} \Delta t / 2<0,
$$

corresponding to the dissipated power

$$
P_{\text {up }}=\lambda \dot{x}^{3} / 2>0 \text {. }
$$

During the downward motion, the external force $F_{0}$ [see Eq. (1b)] performs negative work on a small part of the string whose displacement is $\dot{x} \Delta t / 2$ on the average. The corresponding work is

$$
\Delta W_{\text {down }}=F_{0} \Delta x=F_{0} \frac{\dot{x} \Delta t}{2}=\lambda \dot{x}^{3} \frac{\Delta t}{2}<0,
$$

associated with the dissipated power

$$
P_{\text {down }}=-\lambda \dot{x}^{3} / 2>0,
$$

which, as in the case of the upward motion, is positive because $\dot{x}<0$.

Both downward and upward mechanisms are associated with the fact that a finite force acts on an infinitesimally small element of the system of mass $\lambda|\dot{x}| \Delta t$.

\section{INTEGRATING THE EQUATION OF MOTION}

We introduce the deviation $z=x-x_{0}$ from the equilibrium position $x_{0}=(F-M g) / \lambda g$ where the balloon will eventually stop. In terms of the variable $z$, Eqs. (1a) and (1b) become

$$
\begin{aligned}
& (A+\lambda z) \ddot{z}+(\lambda+\hat{\lambda}) \dot{z}^{2}+B z=0 \quad \text { (upward), } \\
& (A+\lambda z) \ddot{z}+\hat{\lambda} \dot{z}^{2}+B z=0 \quad \text { (downward), }
\end{aligned}
$$

where $A=F / g$ and $B=\lambda g$. Equations (9a) and (9b) can be recast by the change of dependent variable $f=\dot{z}^{2}$ into the form

$$
\begin{aligned}
& (A+\lambda z) f^{\prime}+2(\lambda+\hat{\lambda}) f+2 B z=0 \quad \text { (upward) } \\
& (A+\lambda z) f^{\prime \prime}+2 \hat{\lambda} f+2 B z=0 \quad \text { (downward) }
\end{aligned}
$$

where the prime stands for the derivative with respect to $z$. Equations (10a) and (10b) coincide when the motion direction is reversed, that is, when $\dot{z}=0$. For small oscillations, the terms $z \ddot{z}$ and $\dot{z}^{2}$ are negligible compared to $B z$, so that Eqs. $(9 a)$ and $(9 b)$ reduce to the single harmonic-oscillator equation

$$
\ddot{z}+\Omega^{2} z=0,
$$

where $\Omega=\sqrt{B / A}=g \sqrt{\lambda / F}$. 


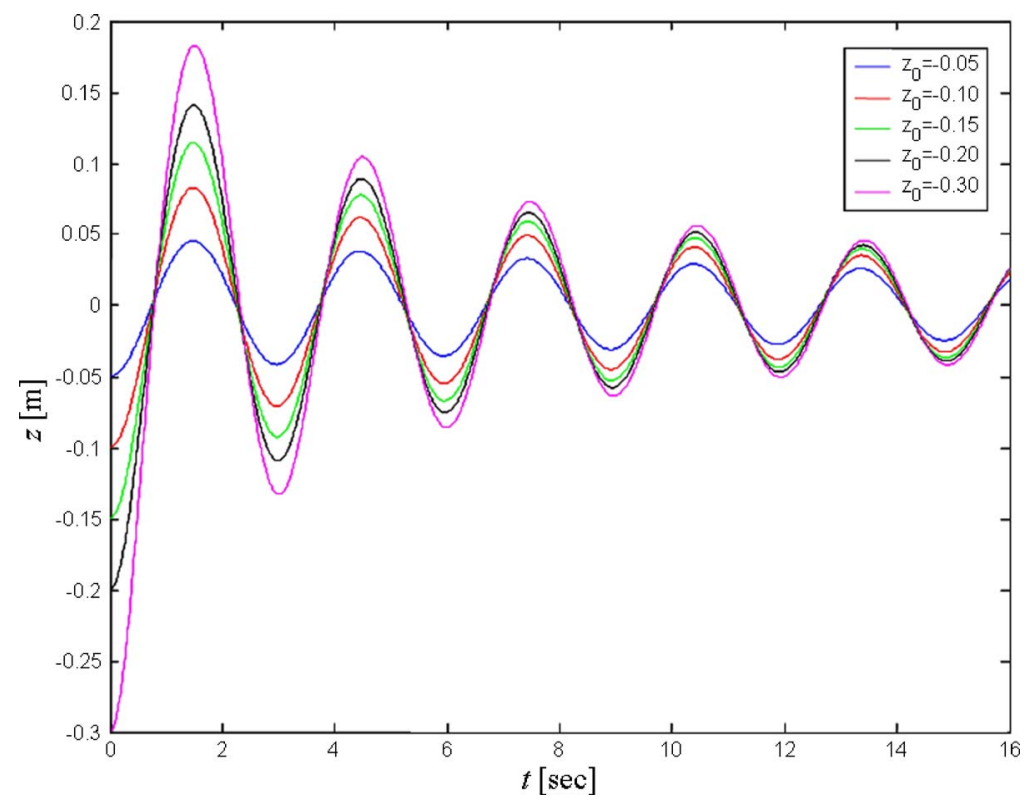

Fig. 2. Simulations of the balloon trajectory $z(t)$ for different initial conditions showing the nonlinear dependence of the oscillations on the amplitude.

Equations (10a) and (10b) can be easily integrated to give $\dot{z}^{2}$ as a function of $z$, but the resulting expression must be analyzed numerically (see Sec. V).

The dynamics of the system presents an unexpected analytical behaviour when the balloon motion inverts its direction (corresponding to $\dot{x}=0$ ), that is, a discontinuity in the fourth time derivative of $x(t)$, as can be checked by successive differentiation of Eqs. (1a) and (1b). This singular feature appears to be a peculiar property of variable-mass dynamics: ordinary discontinuities are associated either with an abrupt change of the propagation medium or with the presence of impulsive forces and result in discontinuities of the first and second derivatives. ${ }^{5}$

\section{NUMERICAL ANALYSIS}

Equations (1a) and (1b) for the balloon-string system has been solved by a fourth-order Runge-Kutta integration algorithm. ${ }^{6}$ The parameters for the simulation were chosen to match those of the experiments (see Sec. VI) with $R$ $=16 \mathrm{~cm}, \lambda=9.8 \mathrm{~g} / \mathrm{m}$, and $\rho_{a}=1.25 \mathrm{~kg} / \mathrm{m}^{3}$. The mass of the balloon was $m=10 \mathrm{~g}$ and the mass of the helium is estimated to be about $10 \mathrm{~g}$ at STP conditions. For these typical dimensions and masses we have (see the following) $\hat{\lambda} / \lambda=\left(\frac{1}{2}\right)$ $\times\left(\rho_{a} / \lambda\right) C A \cong 1$, and hence the two dissipation forces are comparable. (The ratio $\hat{\lambda} / \lambda$ depends strongly on the fluid properties and on the balloon dimensions; $C$ is approximately 0.5 for Reynolds numbers between $10^{2}$ and $10^{5}$ that are typical of our system dynamics. ${ }^{4}$ )

Figure 2 shows numerical simulations of the balloon trajectories for various initial conditions. Note that the upward and downward "half-periods" are not equal and are slightly shorter for the downward motion. The oscillations are nonlinear, that is, the period depends on the amplitude of the oscillations. Although the downward half-period is shorter for larger amplitudes, the successive upward half-period is longer and, as a result, the overall periods of oscillation are almost amplitude independent. Also the amplitude of the os- cillation decrease is larger than that induced by air resistance alone, due to the new dissipation mechanism we have discussed.

Figure 3 depicts the calculated half periods as a function of the initial oscillation amplitude, showing the characteristics of the nonlinear oscillations. As the oscillation amplitude decreases (due to dissipation) the upward and downward half-periods converge to similar values.

\section{A SIMPLE TABLE-TOP EXPERIMENT}

The experimental setup used to study the dynamics of the system is simple. After choosing a balloon with enough buoyancy to push it against the ceiling in the absence of any tail, we tie it to a tail of suitable linear density $\lambda$, so that it eventually reaches a mid-air equilibrium position $x_{0}$. At this point, we have to manually raise or lower the balloon and leave it free to move. The motion of the balloon is then recorded using a digital camcorder. The advantage of using a camcorder is that the pixels can be used to evaluate the position of the balloon. A marker was placed on the balloon (see Fig. 4) and the video signal was analyzed frame by frame to evaluate the position of the marker as a function of time. The pixel data were calibrated by photographing a ruler placed at the same distance from the camcorder as the balloon (see Fig. 4). This calibration method also takes into account the parallax, that is, the relation between the actual resolution of the image and the position of the pixel.

The parameters of the experimental setup are the same as those used in the simulations. Figure 5 shows a comparison of the measured balloon trajectories for the initial conditions $z_{0}=-12.5 \mathrm{~cm}$ (solid line) and $z_{0}=-15.8 \mathrm{~cm}$ (dashed line). The measured period is approximately $3 \mathrm{~s}$, which is in good agreement with the period obtained numerically. A comparison of the experimental results to the simulation reveals some of the predicted trends such as the dependence of the period on the amplitude (larger amplitudes correspond to shorter periods). However, the damping is slightly faster than that predicted by the theory, indicating that there is an addi- 


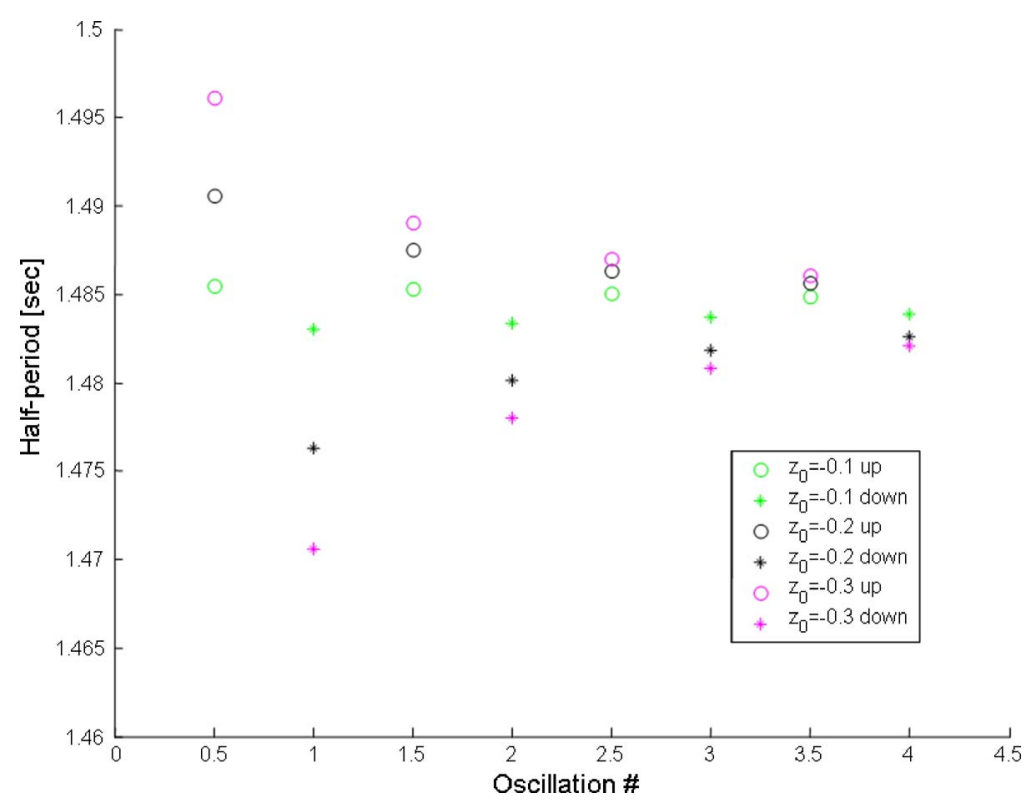

Fig. 3. Calculated half-periods versus the initial amplitude demonstrating the increase/decrease of the upward/downward half-periods for larger oscillation amplitudes.

tional dissipative force not included in our model. The additional dissipation can be reasonably attributed to the tail friction with the floor. In effect, unlike the ideal model, the actual motion of the balloon (and tail) is not restricted, unless special precautions are taken, to the vertical dimension: the lateral motion, due either to the asymmetric shape of the balloon or to the presence of air currents, drags the string on the floor, thus introducing additional dissipation.

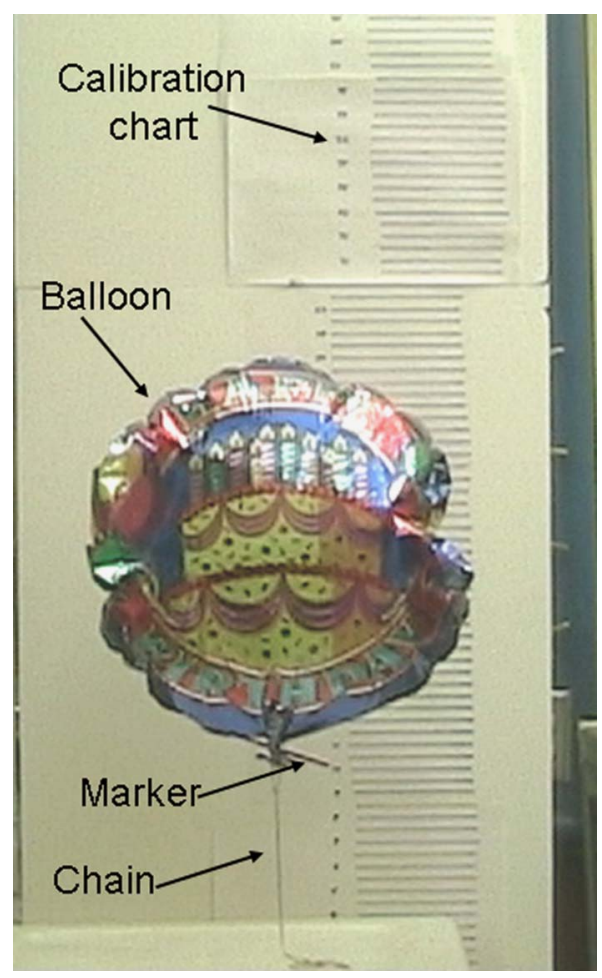

Fig. 4. View of the balloon system under observation.

\section{BEYOND THE PARTY BALLOON}

The party balloon is a particular illustration of a variablemass system. A less accessible example is provided by the system illustrated in Fig. 6 in which gravity and Archimedes' force are replaced by a spring exerting a force $-k\left(x-x_{0}\right)$. When the piston moves toward positive $x$, liquid/gas from the surroundings penetrates the space created within the cylinder and can be assumed to be immediately accelerated to the velocity of the piston (a reasonable assumption if the piston is not moving very fast). Accordingly, the variable fluid-mass within the cylinder is given by $m(t)=A \rho x(t)$, where $\rho$ is the fluid density and $A$ is the piston crosssectional area. We denote the piston mass by $M$ and replace Eqs. (1a) and (1b) by

$$
\begin{aligned}
& -k\left(x-x_{0}\right)=M \ddot{x}+S \rho \dot{x}^{2}+S \rho x \ddot{x}, \quad \dot{x}>0, \\
& -k\left(x-x_{0}\right)=M \ddot{x}+S \rho x \ddot{x}^{2}, \quad \dot{x}<0 .
\end{aligned}
$$

The general properties of the system are qualitatively analogous to those of the balloon, the asymptotic piston position coinciding with the spring rest position $x_{0}$.

The party balloon may be considered as a particular realization of a general class of variable-mass systems. Consider a mechanical system described by a single coordinate $x(t)$ and moving in an arbitrary conservative potential $V$ whose mass varies as a function of space and time. The system can lose mass to the environment in some section of its trajectory and gain mass in other sections. We assume the system environment to be in thermal equilibrium and relatively "cold," that is, the velocity of the environment mass interacting with the system is small compared with that of the system itself. One consequence of this assumption is that when the system gains mass, the added mass is accelerated from zero velocity to the velocity $\dot{x}$ of the system. Also, when the system loses mass, the corresponding kinetic energy is immediately absorbed by the environment. From the point of view of the 


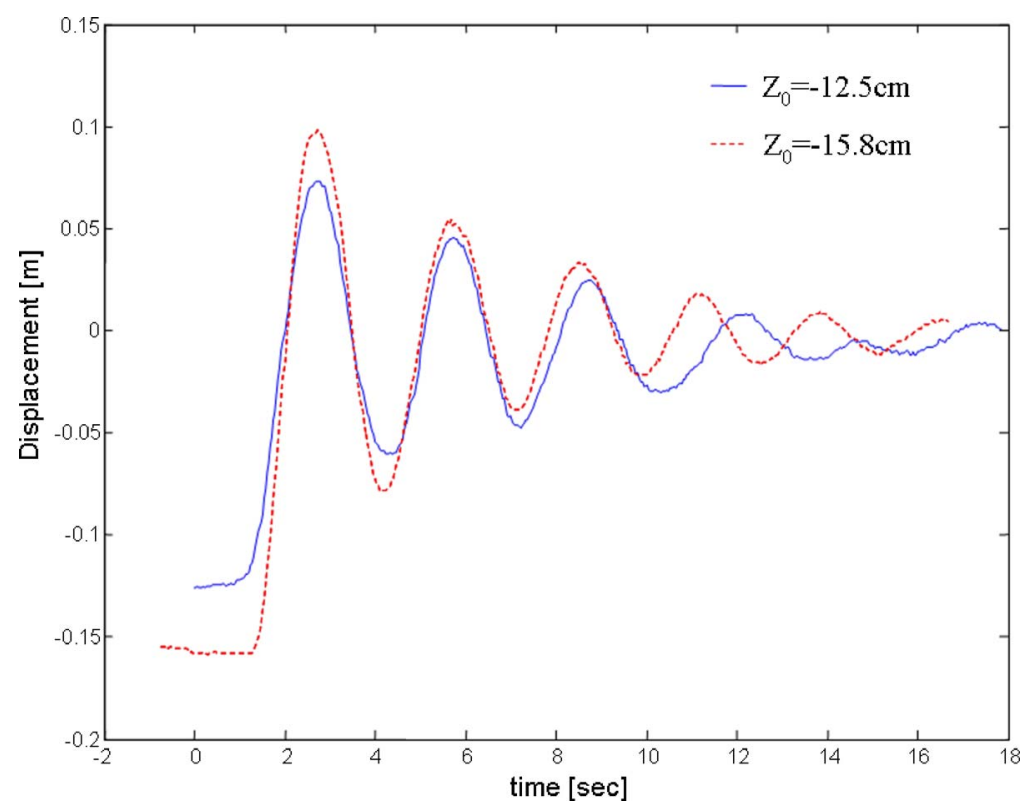

Fig. 5. Measured balloon trajectories for two different initial conditions demonstrating the nonlinear dependence of the system dynamics on the amplitude.

system, this excess mass practically "vanishes," the environment acting as a "mass reservoir." Systems belonging to this class obey the equations of motion

$$
\begin{aligned}
-\frac{d V}{d x}= & \frac{d}{d t}(M \dot{x})=M \ddot{x}+\dot{M} \dot{x}=M \ddot{x}+\frac{\partial M}{\partial x} \dot{x}^{2} \\
& +\frac{\partial M}{\partial t} \dot{x}, \quad \text { for } \dot{M} \geq 0 \\
-\frac{d V}{d x}= & M \ddot{x}, \quad \text { for } \dot{M} \leq 0
\end{aligned}
$$

where $M(x(t), t)$ is the system mass. If $E=\left(\frac{1}{2}\right) M \dot{x}^{2}+V(x)$ is the total mechanical energy of the system, then from Eqs. (14) and (15) it follows that

$$
\frac{d E}{d t}=-\frac{1}{2} \dot{M} \dot{x}^{2}, \quad \text { for } \dot{M} \geq 0,
$$

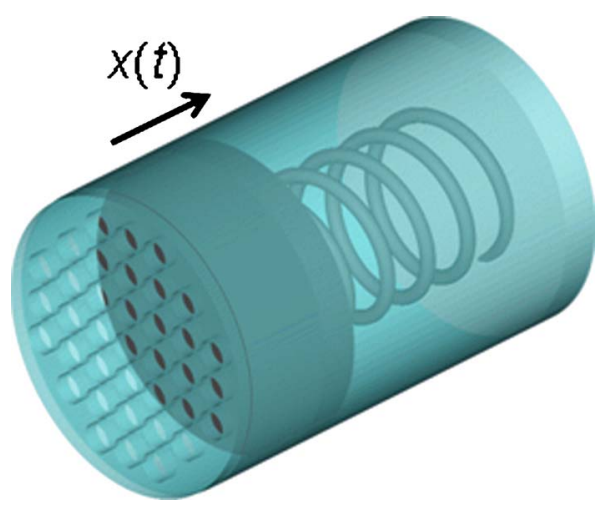

Fig. 6. The unsealed piston system.

$$
\frac{d E}{d t}=\frac{1}{2} \dot{M} \dot{x}^{2}, \quad \text { for } \dot{M} \leq 0 .
$$

As expected, the balloon equations are recovered (apart from the phenomenological contribution due to air resistance which has to be added ad hoc) by setting $M=M(x(t))=M$ $+\lambda x$.

\section{CONCLUSIONS}

A party balloon provides, under special conditions, a straightforward example of a variable mass system. Its dynamic behavior can be understood by means of simple experimental and analytic tools, which makes it an excellent candidate for didactic purposes. In particular, its investigation reveals the emergence of a very general dissipation mechanism that mimics that associated with air resistance without the introduction of any phenomenological coefficient. We hope that both teachers and students may like the light touch introduced in the classroom by the party balloon.

${ }^{a)}$ Author to whom correspondence should be addressed. Electronic mail: kobys@eng.tau.ac.il

${ }^{1}$ R. A. Bachman, "Idealized dynamics of balloon flight," Am. J. Phys. 52, 309-312 (1984).

${ }^{2} \mathrm{~W}$. R. Mellen, "Oscillation of a gas balloon due to a temperature gradient," Am. J. Phys. 58, 781-782 (1990).

${ }^{3} \mathrm{~W}$. T. Achor, "Suspended balloon: An empirical deduction of Archimedes' principle," Am. J. Phys. 28, 166-167 (1960).

${ }^{4}$ L. D. Landau and E. M. Lifshitz, Fluid Mechanics (Pergamon, London, 1959), Chap. 4.

${ }^{5}$ A. S. Ramsey, Dynamics (Cambridge U. P., Cambridge, 1956).

${ }^{6}$ L. F. Shampine, Numerical Solution of Ordinary Differential Equations (Chapman \& Hall, New York, 1994). 